Supporting information for:

\title{
Room-temperature antiferroelectricity in multiferroic hexagonal rare-earth ferrites
}

Jun Kasahara ${ }^{1}$, Tsukasa Katayama ${ }^{1 *}$, Shishin $\mathrm{Mo}^{1}$, Akira Chikamatsu ${ }^{1}$, Yosuke Hamasaki², Shintaro Yasui $^{3}$, Mitsuru Itoh ${ }^{3}$ and Tetsuya Hasegawa ${ }^{1}$

${ }^{1}$ Department of Chemistry, The University of Tokyo, Bunkyo-ku, Tokyo 113-0033, Japan.

${ }^{2}$ Department of Applied Physics, National Defense Academy, Yokosuka 239-8686, Japan.

${ }^{3}$ Laboratory for Materials and Structures, Tokyo Institute of Technology, Midori-ku, Yokohama, 226-8503 Japan.

*Corresponding author: katayama@chem.s.u-tokyo.ac.jp 


\section{Structural characterization}

The in-plane lattice constant of the $h$-DFO film was evaluated using two dimensional XRD $2 \theta$ $\chi$ pattern measurement (Fig. S1). The 114 and 116 diffraction peaks were clearly observed. From the peak positions and the out-of-plane $2 \theta-\theta$ XRD pattern measurement, the $a$ - and $c$-axis lengths were determined to be 6.24 and $11.82 \AA$, respectively.

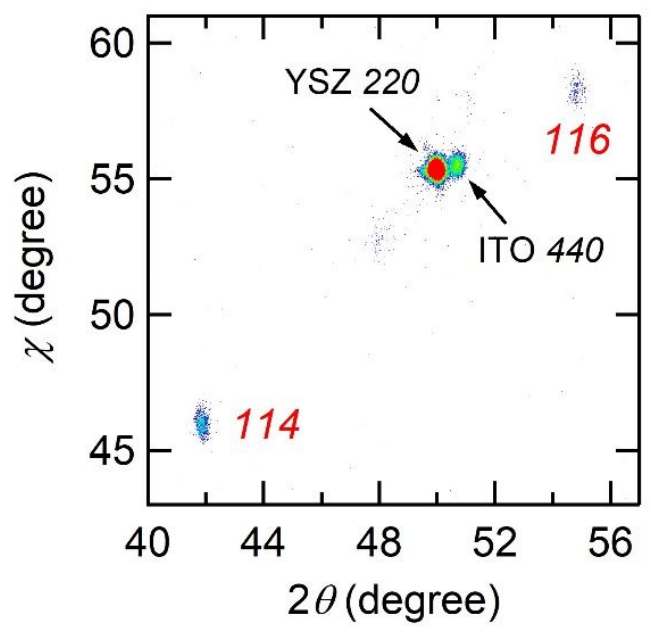

Figure S1. Two dimensional XRD $2 \theta-\chi$ pattern of the $h-\mathrm{DyFeO}_{3}$ film on ITO(111)/YSZ(111) substrate.

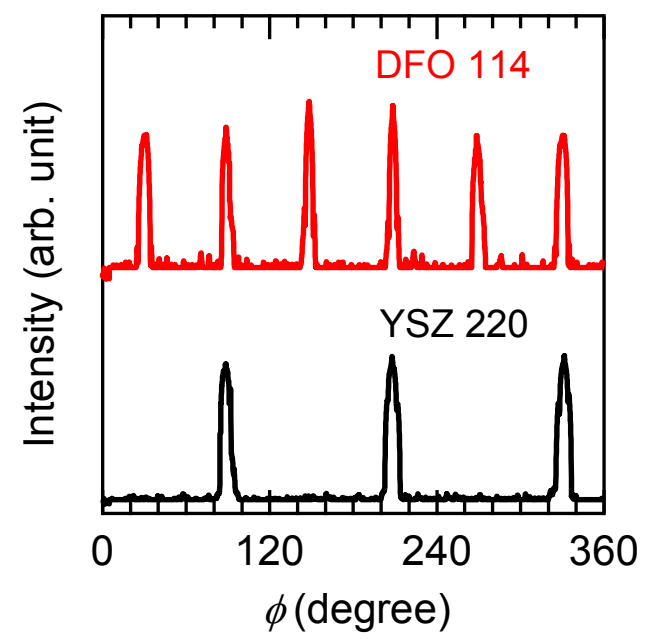

Figure S2. XRD $\phi$-scan of the $h$ - $\mathrm{DyFeO}_{3}$ film on ITO(111)/YSZ(111) substrate. 


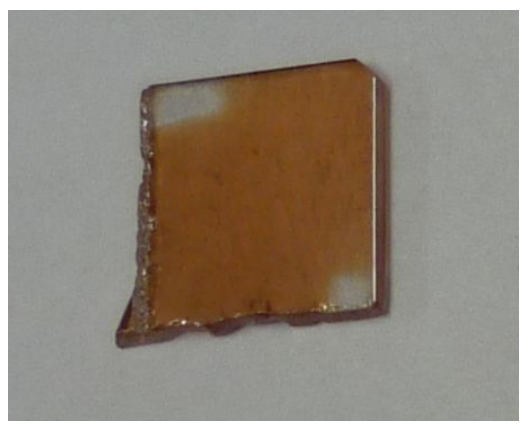

Figure S3. A photograph of the $h$-DFO film on ITO/YSZ substrate. The size is about $5 \mathrm{~mm} \times 5 \mathrm{~mm}$.

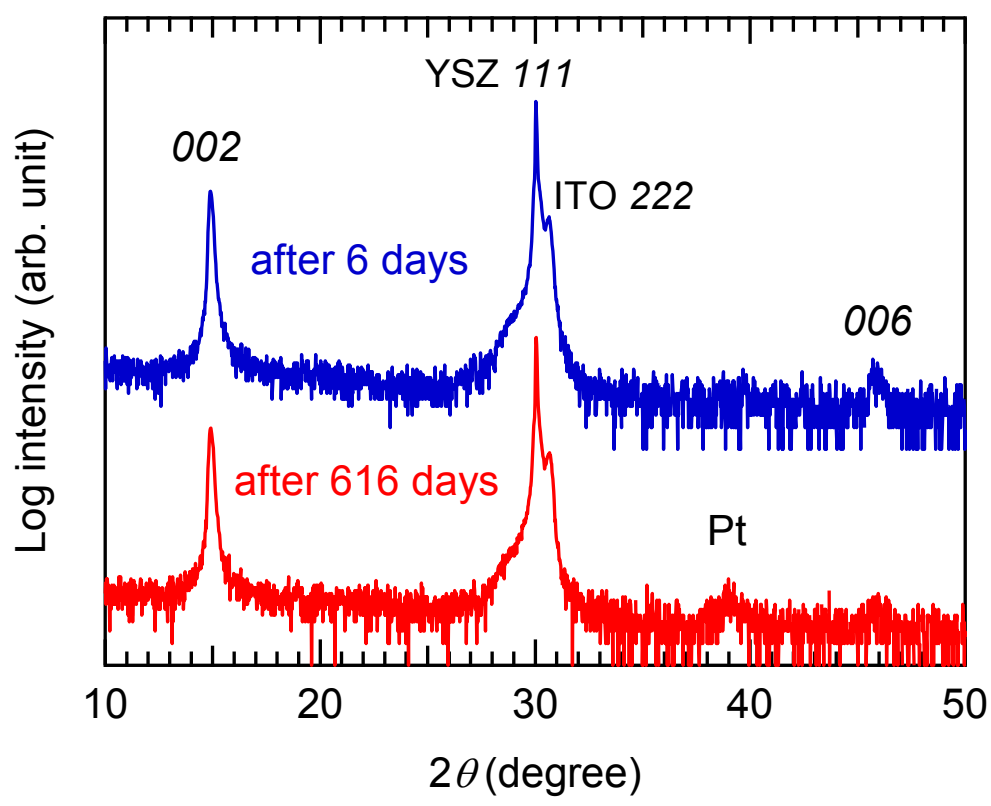

Figure S4. Out-of-plane $2 \theta-\theta$ XRD patterns of the $h$-DFO film measured after 6 and 616 days from the fabrication. 


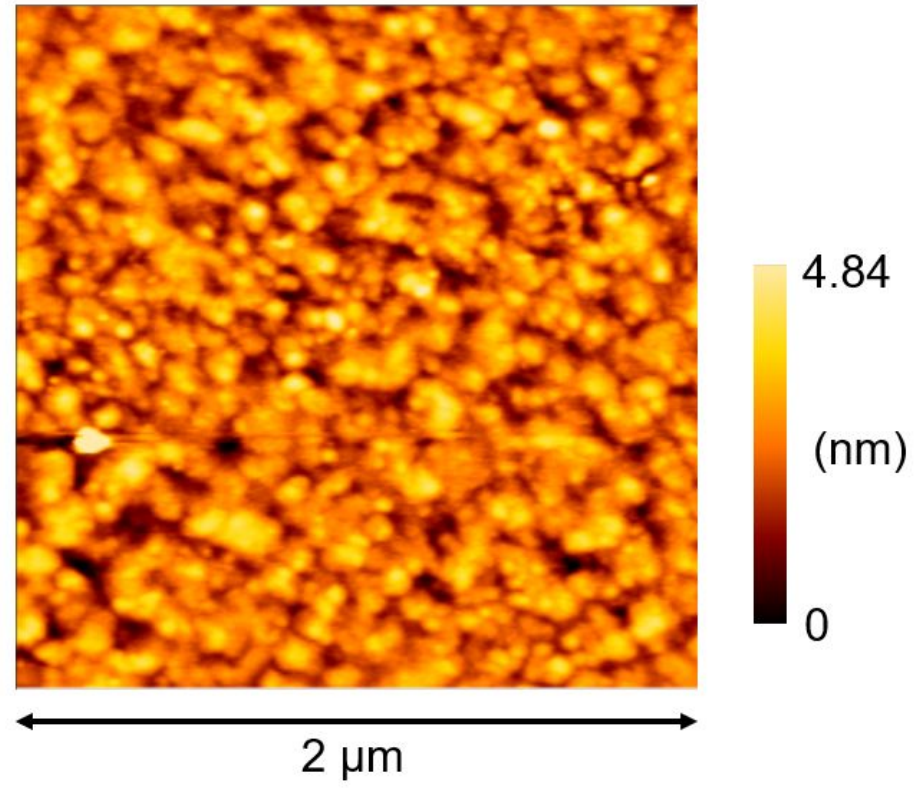

Figure S5. AFM image of the $h$-DFO film.

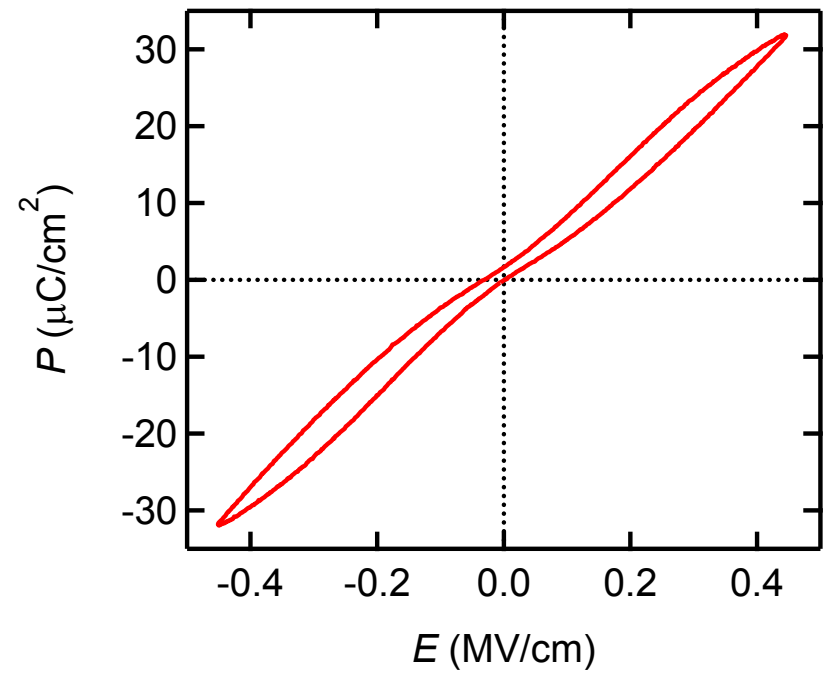

Figure S6. $P-E$ curve of the $h$-DFO film at $100 \mathrm{~K}$ and $10 \mathrm{kHz}$. 

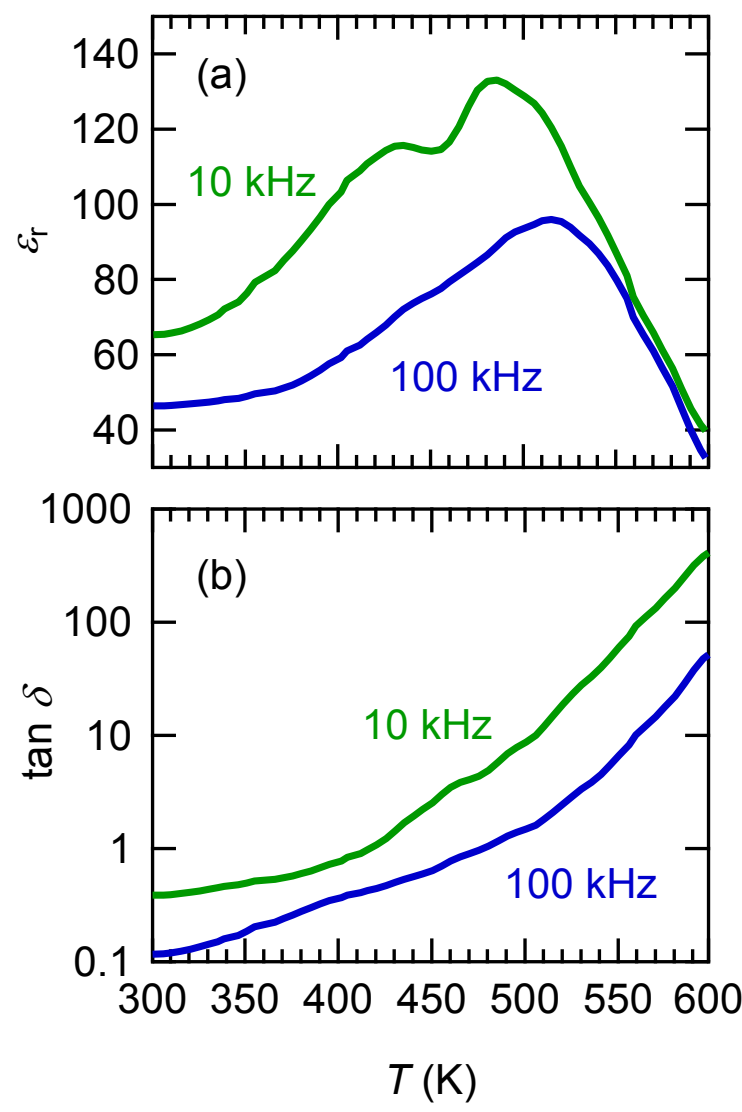

Figure S7. Temperature (T) dependence of (a) permittivity $\left(\varepsilon_{\mathrm{r}}\right)$ and (b) $\tan \delta$ for the $h$-DFO film at 10 and $100 \mathrm{kHz}$. 